\title{
Early identification of patients at risk of acute severe pancreatitis with Systemic Inflammatory Response Syndrome score at admission
}

\section{Laudari U', Thapa $\mathrm{A}^{2}$, Bohara TP3 ${ }^{3}$, Rupakheti $\mathrm{S}^{4}$, Joshi MR}

'Uttam Laudari, Lecturer, Patan Academy of Health Sciences; ${ }^{2}$ Abishek Thapa, Lecturer, KIST Medical College; ${ }^{3}$ Tanka Prasad Bohara, Associate Professor, Kathmandu Medical College; ${ }^{4}$ Shail Rupakheti, Consultant, Star Hospital; ${ }^{5}$ Mukund Raj Joshi, Professor, Kathmandu Medical College, Kathmandu, Nepal.

\begin{abstract}
Background: Clinically, the Systemic Inflammatory Response Syndrome (SIRS) is identified by two or more symptoms including fever or hypothermia, tachycardia, tachypnea and change in blood leukocyte count. The relationship between SIRS symptoms at the time of presentation and severity of pancreatitis is yet to be determined though progression of SIRS in subsequent days has already been correlated.

Objectives: To determine the severity of pancreatitis with SIRS score at the time of admission.

Methodology: A retrospective cohort study of patients admitted to Department of Surgery, Kathmandu Medical College Teaching Hospital (KMCTH) with diagnosis of Acute Pancreatitis (AP) from December 2014 to January 2016 was conducted. Clinical, biochemical and imaging data were collected from the medical record section. Patients with diagnosis of acute pancreatitis as per Revised Atlanta Classification 2012 were included in the study. SIRS score at time of admission was correlated with Modified Marshall scoring system for organ dysfunction. Patients were grouped into severe and nonsevere group. Sensitivity, specificity and predictive values of SIRS score at admission for organ failure were calculated. Results: Among the 41 patients admitted with diagnosis of acute pancreatitis irrespective of cause, the sensitivity of SIRS score $\geq 2$ at admission in predicting severe pancreatitis was $60 \%$, specificity was $20 \%$, positive predictive value was $28 \%$ and negative predictive value was $20 \%$, with p-value of 0.52 and odds ratio of 1.6 (Cl: 0.376-6.808).

Conclusion: SIRS score at admission cannot be solely used in predicting acute severe pancreatitis. Patients can be stratified in resource deficit setting for timely referral to tertiary centre.
\end{abstract}

Key words: Pancreatitis; Severity; Systemic Inflammatory Response Syndrome

DOI: https://doi.org/10.3126/jkmc.v8i2.28171

\section{INTRODUCTION}

A cute pancreatitis (AP) is defined as an acute condition presenting with abdominal pain and is usually associated with raised pancreatic enzyme levels in the blood or urine as a result of pancreatic inflammation'. It occurs in isolated or relapsing events, ranging from mild inflammation to extensive necrosis and liquefaction of pancreas as severe form ${ }^{1,2}$.

Address for correspondence

Dr. Uttam Laudari

Lecturer, Department of Surgery

Patan Academy of Health Sciences, Lagankhel-5, Lalitpur, GPO Box 26500

E-mail: youttam@hotmail.com
The diagnosis is based on abdominal pain; raised pancreatic enzymes (amylase, and/or lipase levels to $>3$ times the upper limit of normal) and imaging test showing features of pancreatitis according to Revised Atlanta Classification 2012 ${ }^{3}$. The most common etiology are alcohol and gall stones ${ }^{1}$. The severity of pancreatitis is defined according to the local and systemic complication. Most of the cases are self-limiting, however mortality of mild diseases are rare, where as in severe pancreatitis, it ranges from $36-50 \%{ }^{3}$.

Severity of AP is assessed by many standardized scoring systems. Most commonly used Scoring system are RANSON's scoring system, Glasgow, Acute Physiology and Chronic Health Evaluation II (APACHE II) and many others ${ }^{4}$. Most scoring system are expensive, time- 
consuming and only suitable for patients admitted in Intensive Care Unit (ICU), and may not be applicable for use in all patients routinely4. Recently Sequential Organ Failure Assessment (SOFA) has been developed and proposed in septic patient in the intensive care setting, and not for routine use in all patients presenting with $A P^{5}$. As such, they are not suitable for stratifying patients at the time of admission or shortly thereafter ${ }^{4}$ and these scoring systems are cumbersome and lots of investigations are required. They are even costly and time consuming to predict the prognosis. Every patient may not be able to afford such long list of investigations and even most of such investigations are not easily available in every hospital.

SIRS is the systemic response to injury where major insults to the host are associated with an overwhelming inflammatory response that, without appropriate and timely intervention, can lead to multiple organ failure and adversely impact patient survival, in the absence of infection, as it does in severe burns, trauma, and pancreatitis ${ }^{6}$. SIRS is defined by the presence of two or more of the following four criteria:

(1) temperature < 36 degree Celsius (96.8 degree Fahrenheit) or $>38$ degree Celsius (100.4 degree Fahrenheit )

(2) heart rate $>90 / \mathrm{min}$

(3) respiratory rate $>20 / \mathrm{min}$, and

(4) white blood cells $\left(<4 \_109 / \mathrm{L}(<4 \mathrm{~K} / \mathrm{mm} 3)\right.$, $>12 \_109(>12 \mathrm{~K} / \mathrm{mm} 3)$ or $10 \%$ bands $^{7}$

In every critical surgical and medical cases, body temperature, heart rate, respiratory rate and total blood count are routinely measured on regular basis. Most of the interventions during management are done according to variability in these parameters. So, simple and easy way of predicting severity of pancreatitis like any other inflammatory condition can be done just using SIRS scoring at admission.

\section{METHODOLOGY}

The study was conducted after receiving the approval letter from Institutional Review committee of Kathmandu Medical College. The entire consecutive patients with diagnosis of Acute Pancreatitis meeting the inclusion criteria were included in the study. The demographic, biochemical, hematological and radiological investigation relevant in diagnosis and stratification of severity of pancreatitis were collected retrospectively from December 2014 to Jan 2016. The diagnosis of AP was made if two of the three criteria were met as per Revised Atlanta classification ${ }^{3}$.
Modified Marshall scoring system was used to define the presence and absence of organ failure (Table 1). A score of $>2$ in one or more of the three organ systems was defined as organ failure. Transient organ failure was defined if any of the involved organ failure lasted $<48$ hours and persistent if $>48$ hours respectively ${ }^{8}$. Severity of Pancreatitis was defined as per Revised Atlanta Classification ${ }^{3}$.

The SIRS score at admission and Modified Marshall scoring after 48 hours were analyzed and patient were stratified for severity according to Revised Atlanta Classification. These patients were grouped into severe and non-severe groups, and also into patients with SIRS score $\geq 2$ or $<2$.

The relation between SIRS scores $\geq 2$ and severity of pancreatitis was evaluated with Fischer Exact Test. The sensitivity, specificity, negative predictive value (NPV) and positive predictive value (PPV) for SIRS score at admission to predict severe pancreatitis was calculated. Odds ratios were reported with 95 confidence intervals and 2-sided $p$-values were reported.

\section{RESULTS}

Total forty one patients were included in the study. One patient had necrotizing pancreatitis at admission and was excluded. The mean age of the patients was 44.51 years and male to female ratio 3.5:1. The mean BMI was $23.58 \mathrm{~kg} / \mathrm{m}^{2}$. The most common cause of AP was biliary $(n=21,53.7 \%)$ followed by alcohol induced $(n=14,34.1 \%)$. Twenty one (51.2\%) of them had SIRS $\geq 2$ at admission. Thirty eight (92.7\%) patients had no any local complication whereas one $(2.4 \%)$ and two $(4.9 \%)$ had acute necrotizing pancreatitis and acute pancreatic fluid collection respectively. Patients were stratified according to Modified Atlanta classification 2012; mild disease $(n=30,73.3 \%)$, moderately severe $(n=1,2.4 \%)$ and severe disease $(n=10,24.3 \%)$ and further grouped into non-severe (mild pancreatitis) and severe group (moderately severe and severe pancreatitis). The most common cause associated with biliary pancreatitis was acute calculus cholecystitis ( $n=5,12.2 \%)$ followed by cholelithiasis $(n=3,7.3 \%)$, choledocholithiasis $(n=2$, $4.9 \%)$ and GB sludge $(n=2,4.9 \%)$. Sixty one percent of the cases had no any associated diseases.

The sensitivity for SIRS $\geq 2$ at admission for severity was found to be $60 \%$, specificity $20 \%$, PPV $28 \%$, NPV $20 \%$ and odds ratio of 1.6 (Cl $0.376-6.808 \%)$. ROC curve yielded an AUC of $0.56\{95 \%$ confidence interval $(0.409-0.748)\}$ with $\mathrm{p}$-value 0.719 . The prevalence of severe pancreatitis among patients with SIRS score $0,1,2,3$ and 4 were $25 \%$, $17 \%, 8.6 \%, 33.33 \%$ and $100 \%$ respectively. 
Table 1: Modified Marshall Scoring System for organ dysfunction ${ }^{3}$

\begin{tabular}{|c|c|c|c|c|c|}
\hline \multirow{2}{*}{ Organ/System } & \multicolumn{5}{|c|}{ Score } \\
\hline & 0 & 1 & 2 & 3 & 4 \\
\hline Respiratory( $\mathrm{PaO} 2 /$ Fio2) & $>400$ & $301-400$ & $201-300$ & $101-200$ & $<101$ \\
\hline $\begin{array}{l}\text { Renal* } \\
\text { Serum Creatinine (mg/dl) }\end{array}$ & $<1.4$ & $1.4-1.8$ & $1.9-3.6$ & $3.6-4.9$ & $>4.9$ \\
\hline $\begin{array}{l}\text { Cardiovascular } \\
\text { (systolic blood pressure, } \mathrm{mm} \mathrm{Hg} \text { ) }\end{array}$ & $>90$ & $\begin{array}{l}<90 \text { fluid } \\
\text { responsive }\end{array}$ & $\begin{array}{l}<90, \text { not fluid } \\
\text { responsive }\end{array}$ & $<90, \mathrm{pH}<7.3$ & $<90, \mathrm{pH}<7.2$ \\
\hline
\end{tabular}

For non-ventilated cases (Fraction of inspired oxygen) $\mathrm{FiO}_{2}$ can be estimated from:

\begin{tabular}{ccc} 
Supplement oxygen & FiO2 (\%) \\
Room air & 21 \\
2 & 25 \\
4 & 30 \\
$6-8$ & 40 \\
$9-10$ & 50 \\
\hline
\end{tabular}

A score of 2 or more in any system defines the presence of organ failure.

${ }^{*}$ A score for patients with pre-existing chronic renal failure depends on the extent of further deterioration of baseline renal function. No formal correction exists for a baseline serum creatinine $\geq 134 \mu \mathrm{mol} / \mathrm{l}$ or $\geq 1.4 \mathrm{mg} / \mathrm{dl}$.

tOff inotropic support

\section{DISCUSSION}

This study showed that majority of patients with AP had SIRS $\geq 2$ at admission. It was definitely associated with poor prognosis than patients without SIRS at admission, however the sensitivity for SIRS at admission was far too less as compared to previous study?

Grover et al. conducted similar study in 50 pediatric patients where they found two or more SIRS criteria at admission had longer hospital stay (median 8 days, 4-13 days) as compared to patients without SIRS (median 3 days, 1-1.5days) which was statistically significant. Also $32 \%$ patients with SIRS at admission had ICU admission compared to $4 \%$ of those without SIRS ${ }^{10}$. This study was conducted among adult patients and outcome analyzed was only for severity of disease after 48 hours and all patients with SIRS score 4 had severe pancreatitis.

Singh et al. in 2009 found that $62 \%$ of patients had SIRS on day one, with $85-100 \%$ sensitivity in predicting severity, and that patient without SIRS had NPV of 98$100 \%$ for severity ${ }^{9}$. Twenty one (51.2\%) of our patients had SIRS $\geq 2$ at admission. The sensitivity and specificity in our study was also less as compared to the study by Singh et al. with magnitudes $60 \%$ and $20 \%$ respectively. The disparity in this finding is probably due to low sample size. Also Singh et al. had taken average SIRS at day one, whereas we studied SIRS at presentation in emergency department, which may be influenced by pain, anxiety and pre-treatment at other centres.
Singh et al. also found that patients with higher number of SIRS on day one predicted the severity, whereas our study was not able to find the strong association between the number of SIRS criteria and severity of pancreatitis as of previous study, but the prevalence of severity was higher in patients among SIRS score 3 and 4. The sensitivity of $S I R S \geq 3$ for severity at admission was $20 \%$, specificity being $6.8 \%$, PPV of $50 \%$ and NPV $78.37 \%$. This could also be due to the change in SIRS variable after admission in hospital. So, single recording of SIRS criteria at admission cannot be solely used to predict severity, however in resources deficit setting it can help to stratify reasonable number of patient.

As with any ongoing inflammatory markers in body, SIRS component are raised in AP also. Transient and persistent SIRS had poor outcome in $\mathrm{AP}^{11}$. Our study found that SIRS score at admission cannot be solely used as a screening tool but can definitely stratify sixty percent of patients at presentation. This study highlights that SIRS at admission can be taken into account while waiting for other prognostic scores which are costly, time consuming and not readily available everywhere.

There were few limitations in our study. First, it is a single centre study with small sample size, so the results cannot be generalized. Second, the study centre is a tertiary centre where number of patients may already have received primary care at other centres. Third, each variable of SIRS may be influenced by other associated 
inflammatory and infectious conditions which may have gone unnoticed.

\section{CONCLUSION}

SIRS is response of body to inflammatory and infectious insult in body. SIRS score at admission can certainly stratify fair number of patients and can be used as a tool in resource deficit setting. SIRS score at admission alone cannot predict severity, continuous SIRS monitoring is required.

\section{REFERENCES}

1. Bailey, H., Love, R. J. M. N., Mann, C. V., \& Russel, R.C.G. Bailey and Love's Short Practice of Surgery. 25th ed.Edward Arnold;c 2008. Chapter 28, The Pancrease: p. $1138-1146$

2. Gupta K, Wu B. In the Clinic. Acute Pancreatitis. Ann Intern Med. 2010 Nov;153(9):1-16. [PubMed]

3. Banks PA, Bollen TL, Dervenis C, Gooszen HG, Johnson CD, Sarr MG et al. Classification of acute pancreatitis-2012: revision of the Atlanta classification and definitions by international consensus. British Medical Journal [Internet]. 2013 Jan [cited 2014 Jul 10];62(1):102-11. [DOI]

4. Suppiah A, Malde D, Arab T, Hamed M, Allgar V, Smith $A M$ et al. The prognostic value of the neutrophillymphocyte ratio (NLR) in acute pancreatitis: identification of an optimal NLR. J Gastrointest Surg [Internet]. 2013 Apr [cited 2014 Jul 15];17(4):675-81. [PubMed]

5. Singer $M$, Deutschman CS, Seymour CW, Shankar-Hari M, Annane D, Bauer M et al. The Third International Consensus Definitions for Sepsis and Septic Shock (Sepsis-3).JAMA [Internet] 2016 Feb;315(8):801-10. [PubMed]

6. Brunicardi FC, Andersen DK, Billiar TR, Dunn DL John G. Hunter R. Schwartz's Principles of Surgery. $8^{\text {th }}$ ed. The McGraw-Hill Companies;c2004. Chapter 33, Pancreas: p. 2000

7. Bone RC, Balk RA, Cerra FB, Dellinger RP, Fein AM, Knaus WA et al. Definitions for sepsis and organ failure and guidelines for the use of innovative therapies in sepsis. The ACCP/SCCM Consensus Conference Committee. American College of Chest Physicians/Society of Critical Care Medicine. 1992. Chest. 1992 Jun; 101(6):1644-55 [PubMed]

8. Banks $\mathrm{P}$ a, Freeman ML. Practice guidelines in acute pancreatitis. Am J Gastroenterol [Internet]. 2006 Oct [cited 2014 Jul 10];101(10):2379-400. [PubMed]

9. Singh VK, Wu BU, Bollen TL, Repas K, Maurer R, Mortele $\mathrm{KJ}$ et al. Early systemic inflammatory response syndrome is associated with severe acute pancreatitis. Clin Gastroenterol Hepatol [Internet]. 2009 Nov [cited 2014 Sep 20];7(11):1247-51. [PubMed]

10. Grover AS, Kadiyala V, Banks PA, Grand RJ, Conwell DL, Lightdale JR. The Utility of the Systemic Inflammatory Respsonse Syndrome Score on Admission in Children With Acute Pancreatitis. Pancreas [Internet].2016;106-9. [DOI]

11. Lee W, Huang J, Chuang W. Outcome assessment in acute pancreatitis patients. Kaohsiung J Med Sci [Internet]. 2013;29(9):469-77. [DOI] 